\title{
Topological and algebraic genericity of divergence and universality
}

\author{
by \\ FRÉDÉRIC BAYART (Bordeaux)
}

\begin{abstract}
We give general theorems which assert that divergence and universality of certain limiting processes are generic properties. We also define the notion of algebraic genericity, and prove that these properties are algebraically generic as well. We show that universality can occur with Dirichlet series. Finally, we give a criterion for the set of common hypercyclic vectors of a family of operators to be algebraically generic.
\end{abstract}

1. Introduction. This paper deals with the pathological behavior of certain series. Its origin goes back to the following observation of du BoisReymond (1873):

There exists a continuous function whose Fourier series diverges at a point.

Later, this property has been proved to be topologically generic in the following sense: it holds for a dense $G_{\delta}$ subset of $C(\mathbb{T})$, the Banach space of continuous functions on the unit circle $\mathbb{T}$. We also say that this property is quasi-sure, or that it holds quasi-everywhere. Recall that in a Baire space, countable intersections of dense open sets are big sets: they are analogues of sets of probability one in probability spaces.

Many other examples of divergence of Fourier series are known (due to Kolmogorov, Fefferman,...) and some of them have a generic version. We refer to the recent survey of J.-P. Kahane ([Ka]) for precise statements. In Section 2, we give a "proving machine theorem" which allows us to obtain the genericity automatically from the existence of one divergent series. Thus, we recover some known results, and discover some apparently new ones (in the case of Fefferman's divergence theorem, or of some recent results on divergence of Dirichlet series).

We also introduce another type of genericity: a property on a Banach space $X$ is said to be algebraically generic if it holds for every non-zero vector

2000 Mathematics Subject Classification: Primary 46B25, 47A16.

Key words and phrases: hypercyclicity, universal family, Dirichlet series, Fourier series, divergence, quasi-sure property. 
of a dense subspace of $X$. Theorem 3 below asserts that the divergence of Fourier series is also an algebraically generic phenomenon.

Sometimes, limiting processes may diverge in a maximal way: this is linked with the phenomenon of universality. We restrict ourselves to the notion of universal series: if $\left(a_{n}\right)_{n \in \mathbb{N}}$ is a sequence of complex numbers, and $\left(x_{n}\right)_{n \in \mathbb{N}}$ is a sequence in a topological vector space $X$, the series $\sum_{n=0}^{\infty} a_{n} x_{n}$ is said to be universal if the sequence of its partial sums $\left(\sum_{n=0}^{N} a_{n} x_{n}\right)_{N \geq 0}$ is dense in $X$. Surprisingly, a great number of universal objects have been discovered: we refer to the survey of K.-G. Grosse-Erdmann [Gre], and just give a recent result of $\mathrm{V}$. Nestoridis ([Ne]):

There exists a Taylor series $S=\sum_{n=0}^{\infty} c_{n} z^{n}$, convergent in the unit disk $\mathbb{D}$, such that, given any compact set $K$ which does not separate the plane and which satisfies $K \cap \mathbb{D}=\emptyset$, and any function $g$ continuous on $K$ and analytic in the interior of $K$, there exists a sequence of partial sums of $S$ which converges to $g$ uniformly on $K$.

If one allows the sequence $\left(a_{n}\right)$ to be in a Baire subset $Y \subset \mathbb{C}^{\mathbb{N}}$, one could ask if the existence of a universal series with coefficients in $Y$ implies the topological genericity of this property in $Y$. Indeed, in the literature, for many examples, universality appears as a generic phenomenon. For instance, quasi-all Taylor series in $H(\mathbb{D})$ are universal in the sense of Nestoridis. Theorem 4 below shows that under some natural assumptions on $Y$, one has a topological zero-one law: either there does not exist a universal series $\sum a_{n} x_{n}$ with $\left(a_{n}\right) \in Y$ (the vectors $x_{n}$ being kept fixed), or for quasi-all sequences $\left(a_{n}\right) \in Y$, the series $\sum a_{n} x_{n}$ is universal. We also give in Section 3 a generalization of the Nestoridis Theorem to Dirichlet series.

For $Y$ a linear space, the notion of universality is of course connected with that of hypercyclicity. Recall that if $X$ and $Y$ are two topological vector spaces, a sequence $\left(T_{N}\right)$ of continuous linear mappings $T_{N}: Y \rightarrow X$ is called hypercyclic provided there exists a vector $y$, called a hypercyclic vector, whose orbit $\left\{T_{N} y ; N \in \mathbb{N}\right\}$ is dense in $X$. Now, if $Y$ is a sequence space, one can define

$$
T_{N}: Y \rightarrow X, \quad\left(a_{n}\right) \mapsto \sum_{n=0}^{N} a_{n} x_{n} .
$$

So, the existence of a universal series is equivalent to the hypercyclicity of $\left(T_{N}\right)$. If $Y$ is a Baire space, the topological genericity of hypercyclic vectors and therefore of universal series is well known (see for instance [Gre]). Moreover, L. Bernal-González gave in [Be, Thm. 3] a sufficient condition for the algebraic genericity of the set of hypercyclic vectors. We will show that this sufficient condition is always satisfied for universal series. 
Recently, the notion of common hypercyclicity has drawn attention ([AG], [CS], [Ba1], [Ba2]): given a family $\left(T_{N, \lambda}\right)_{N \in \mathbb{N}, \lambda \in I}$ of operators $Y \rightarrow X$ such that, for each $\lambda \in I$, the sequence $\left(T_{N, \lambda}\right)_{N \in \mathbb{N}}$ is hypercyclic, does there exist a common hypercyclic vector, that is, a vector $x$ which is hypercyclic for each sequence $\left(T_{N, \lambda}\right)_{N \in \mathbb{N}}$ ? In particular, G. Costakis and M. Sambarino give in [CS] a sufficient condition for the set of common hypercyclic vectors to be topologically generic. In Section 4, we give a similar condition for the set of common hypercyclic vectors to be algebraically generic.

\section{Divergent series}

2.1. A criterion for topological genericity. Our first result asserts that if a limiting process is unboundedly divergent at one point for one function, it is divergent at quasi-all points for quasi-all functions.

Theorem 1. Let $X$ be a Banach space, and $G$ be a complete metrizable abelian group. Let $\left(T_{t}\right)_{t \in G}$ be a continuous group of operators acting on $X$, each of them being an isometry. Let $\left(\varphi_{n}\right)$ be a sequence of continuous linear forms on $X$, and define, for $f \in X, t \in G, \varphi_{n}(f, t)=\varphi_{n}\left(T_{t} f\right)$. Suppose that (H1) for all $M>0$ and $N>0$, there exist $n \geq N$ and $g \in X$ such that $\|g\| \leq 1$ and $\left|\varphi_{n}(g, 0)\right|>M$.

Then for quasi-all functions $f \in X$, the sequence $\left(\varphi_{n}(f, t)\right)$ is unboundedly divergent for quasi-all $t \in G$.

Remark. Hypothesis (H1) is for example an easy consequence of the following one: There exists $g \in X$ such that the sequence $\left(\varphi_{n}(g, 0)\right)$ is unboundedly divergent.

Proof. For $M, N \in \mathbb{Z}^{+}$, set

$$
A(M, N)=\bigcup_{n \geq N}\left\{(f, t) \in X \times G ;\left|\varphi_{n}(f, t)\right|>M\right\} .
$$

This set is clearly open. Let us show that it is dense. We fix $\left(f, t_{0}\right) \in X \times G$ and $\varepsilon>0$ as small as we wish. Let $g \in X$ be a function given by hypothesis (H1) satisfying

$$
\|g\| \leq 1 \text { and }\left|\varphi_{n}(g, 0)\right|>M / \varepsilon \text { for } n \geq N .
$$

Suppose for a moment that the following two inequalities are simultaneously true:

$$
\left|\varphi_{n}\left(T_{t_{0}} f+\varepsilon g, 0\right)\right|<M, \quad\left|\varphi_{n}\left(T_{t_{0}} f-\varepsilon g, 0\right)\right|<M .
$$

By the triangle inequality, one deduces $\left|\varphi_{n}(2 \varepsilon g, 0)\right|<2 M$, which is false. Therefore we can assume for instance that

$$
\left|\varphi_{n}\left(T_{t_{0}} f+\varepsilon g, 0\right)\right|>M
$$


This implies

$$
\left|\varphi_{n}\left(f+\varepsilon T_{-t_{0}} g, t_{0}\right)\right|>M .
$$

Thus, $\left(f+\varepsilon T_{-t_{0}} g, t_{0}\right) \in A(M, N)$, and $\left(f+\varepsilon T_{-t_{0}} g, t_{0}\right)$ can be chosen arbitrarily close to $\left(f, t_{0}\right)$. This implies that $\bigcap_{M, N \in \mathbb{Z}^{+}} A(M, N)$ is residual, and if $(f, t)$ is in this set, the series $\left(\varphi_{n}(f, t)\right)$ is unboundedly divergent. The Kuratowski-Ulam Theorem, as employed in [Ka], gives the conclusion.

EXAMPLE. Theorem 1 could be applied to prove the topological genericity of du Bois-Reymond's example (see [Ka]). We prefer to give a less standard (and apparently new) result for a summability method. A series $\sum_{\text {if }} u_{n}$ is said to be summable by Borel's method, or B-summable, to sum $S$

$$
B(\lambda)=e^{-\lambda} \sum_{n=0}^{\infty} \frac{s_{n} \lambda^{n}}{n !} \rightarrow S \quad \text { as } \lambda \rightarrow \infty,
$$

where $s_{n}=u_{0}+\cdots+u_{n}$. Moore (see [Zyg, p. 314]) proved the existence of a continuous function $f$ on $\mathbb{T}$ whose Fourier series at 0 is not $B$-summable (the divergence being unbounded). Theorem 1 implies that, for quasi-all functions $f \in C(\mathbb{T})$ and quasi-every $t$ in $\mathbb{T}$, the Fourier series of $f$ at $t$ is not $B$-summable.

Indeed, let $X$ be $C(\mathbb{T})$, and $G=\mathbb{T}$. The action of $G$ on $X$ is the translation

$$
T_{e^{i t}} f(z)=f\left(e^{i t} z\right) .
$$

The linear forms

$$
\varphi_{N}(f)=e^{-N} \sum_{n=0}^{\infty} s_{n} \frac{N^{n}}{n !},
$$

where $s_{n}$ is the $n$th partial sum of the Fourier series of $f$, evaluated at the point 0 , satisfy the assumptions of Theorem 1 .

If the limiting process is everywhere unboundedly divergent, everywhere unbounded divergence is also topologically generic:

Theorem 2. Let $X$ be a Banach space, and $E$ be a $\sigma$-compact topological space. Suppose that, for each $t \in E$ and each $n \geq 0$, a linear form $\varphi_{n}(t, \cdot)$ is given such that $\varphi_{n}: X \times E \rightarrow \mathbb{C}$ is continuous. For $g \in X$ and $t \in E$, set

$$
\delta_{N}(g, t)=\sup _{n>N}\left|\varphi_{n}(g, t)-\varphi_{N}(g, t)\right| .
$$

Suppose that

(H2) for all $M, N>0$, and each compact subset $K$ of $E$, there exists $g \in X$ such that $\|g\| \leq 1$ and $\delta_{N}(g, t)>M$ for every $t \in K$.

Then for quasi-all $f \in X$, the sequence $\left(\varphi_{n}(f, t)\right)_{n \geq 0}$ is unboundedly divergent for each $t \in E$. 
REMARK. In many situations, (H2) will follow from the stronger assumption

$\exists g \in X$ such that $\forall t \in E,\left(\varphi_{n}(g, t)\right)$ is unboundedly divergent.

Proof. Write $E=\bigcup_{k=1}^{\infty} X_{k}$, where $\left(X_{k}\right)$ is a non-decreasing sequence of compact subsets of $E$. We shall construct an increasing sequence $\left(N_{k}\right)_{k \geq 0}$ of integers and a sequence $\left(g_{k}\right)_{k \geq 1}$ of vectors in $X$ such that

(1) $\forall t \in X_{k}$,

$$
\sup _{N_{k-1}<n \leq N_{k}}\left|\varphi_{n}\left(g_{k}, t\right)-\varphi_{N_{k-1}}\left(g_{k}, t\right)\right| \geq k, \quad\left\|g_{k}\right\| \leq 1 .
$$

Take $N_{0}=0$. We assume that $N_{0}, \ldots, N_{k-1}, g_{1}, \ldots, g_{k-1}$ have been defined, and describe how to choose $N_{k}$ and $g_{k}$. Let $g_{k}$ be given by (H2) for $M=k$, $N=N_{k-1}, K=X_{k}$, and let

$$
\delta_{N}^{*}(t)=\sup _{N_{k-1}<n \leq N}\left|\varphi_{n}\left(g_{k}, t\right)-\varphi_{N_{k-1}}\left(g_{k}, t\right)\right| .
$$

The continuous functions $\left(\delta_{N}^{*}(t)+1\right)^{-1}$ decrease pointwise to 0 , and Dini's Theorem implies the existence of $N>N_{k-1}$ such that $\left(\delta_{N}^{*}(t)+1\right)^{-1} \leq$ $(k+1)^{-1}$ for each $t \in X_{k}$. Just take $N_{k}=N$ to get (1). Now, for $M, k \in \mathbb{Z}^{+}$, set

$$
A(M, k)=\bigcup_{l \geq k}\left\{f \in X ; \forall t \in X_{k}, \sup _{N_{l-1}<n \leq N_{l}}\left|\varphi_{n}(f, t)-\varphi_{N_{l-1}}(f, t)\right|>M\right\} .
$$

Clearly, $A(M, k)$ is open. Moreover, it is dense: if $f_{0} \in X$ and $\varepsilon>0$ is arbitrarily small, there exists $l \geq k$ such that

$$
\forall t \in X_{k}, \quad \sup _{N_{l-1}<n \leq N_{l}}\left|\varphi_{n}\left(g_{l}, t\right)-\varphi_{N_{l-1}}\left(g_{l}, t\right)\right|>\frac{M}{\varepsilon} .
$$

As in the proof of Theorem 1, either $f_{0}+\varepsilon g_{l}$ or $f_{0}-\varepsilon g_{l}$ belongs to $A(M, k)$. Therefore $\bigcap_{M, k} A(M, k)$ is quasi-sure, and for any $f \in \bigcap_{M, k} A(M, k)$ and any $t \in E,\left(\varphi_{n}(f, t)\right)$ is unboundedly divergent.

Examples. (1) Let $X=L^{1}(\mathbb{T}), E=\mathbb{T}$, and $\varphi_{N}(f, t)=\sum_{|n| \leq N} \widehat{f}(n) e^{i n t}$. A famous result of Kolmogorov (see [Ka] or [Zyg] for a proof) asserts that there exists a function $f \in L^{1}(\mathbb{T})$ such that, for each $t \in \mathbb{T},\left(\varphi_{n}(f, t)\right)$ is unboundedly divergent. This property is topologically generic.

(2) Let $X=L^{2}\left(\mathbb{T}^{2}\right), E=\mathbb{T}^{2}$. Denote by $S_{M, N} f(t, u)$ the Fourier series of $f \in L^{1}\left(\mathbb{T}^{2}\right)$ :

$$
S_{M, N}(f)(t, u)=\sum_{|m| \leq N,|n| \leq N} \widehat{f}(m, n) e^{i m t} e^{i n u} .
$$

Fefferman gives in $[\mathrm{Fe}]$ an example of $f \in L^{2}\left(\mathbb{T}^{2}\right)$ such that, for each $(t, u) \in \mathbb{T}^{2}$,

$$
\limsup _{M, N \rightarrow \infty}\left|S_{M, N} f(t, u)\right|=\infty \text {. }
$$


More precisely, his construction implies the existence of two increasing sequences $\left(M_{k}\right)$ and $\left(N_{k}\right)$ of integers such that

$$
\left|S_{M_{k}, N_{k}} f(t, u)\right| \geq k
$$

for every $(t, u) \in \mathbb{T}^{2}$. By setting $\varphi_{k}(g,(t, u))=S_{M_{k}, N_{k}} g(t, u)$ and by applying Theorem 2, we see that Fefferman's example is topologically generic in $L^{2}\left(\mathbb{T}^{2}\right)$.

(3) Let us now turn to the case of $\mathcal{H}^{\infty}$, the space of Dirichlet series $f(s)=\sum_{n=1}^{\infty} a_{n} n^{-s}$, with convergence and boundedness of $f$ in the halfplane $\mathbb{C}_{+}=\{s \in \mathbb{C} ; \Re(s)>0\} ; \mathcal{H}^{\infty}$ is a Banach space with the norm

$$
\|f\|_{\infty}=\sup \left\{|f(s)| ; s \in \mathbb{C}_{+}\right\} .
$$

In $[\mathrm{BKQ}]$, it is proved that there exists a Dirichlet series $f(s)=\sum_{n=1}^{\infty} a_{n} n^{-s}$ $\in \mathcal{H}^{\infty}$ such that $\sum_{n=1}^{\infty} a_{n} n^{i t}$ diverges for each $t \in \mathbb{R}$. In the course of the proof, we build a sequence of Dirichlet polynomials $Q_{k}(s)=\sum_{n=1}^{N_{k}} a_{n}(k) n^{-s}$ and a sequence of intervals $X_{k}$ such that:

$$
\begin{gathered}
\left\|Q_{k}\right\|_{\infty} \stackrel{k \rightarrow \infty}{\longrightarrow} 0, \quad X_{k} \subset X_{k+1}, \quad \bigcup_{k=1}^{\infty} X_{k}=\mathbb{R}, \\
\forall t \in X_{k}, \quad \sup _{1 \leq l \leq N_{k}}\left|\sum_{n=1}^{l} a_{n}(k) n^{i t}\right| \geq \delta,
\end{gathered}
$$

where $\delta>0$ is an absolute constant. We may apply Theorem 2: for quasiall Dirichlet series $f(s)=\sum_{n=1}^{\infty} a_{n} n^{-s} \in \mathcal{H}^{\infty}, \sum_{n=1}^{\infty} a_{n} n^{i t}$ diverges unboundedly for each $t \in \mathbb{R}$. Observe that in [BKQ], we obtained $f(s)=$ $\sum_{n=1}^{\infty} a_{n} n^{-s} \in \mathcal{H}^{\infty}$ such that $\sum_{n=1}^{\infty} a_{n} n^{i t}$ was boundedly divergent on $\mathbb{R}$.

REMARK. The topological genericity of divergent series $\sum \varepsilon_{n} f_{n}$, where $\left(\varepsilon_{n}\right)$ runs over all choices of signs $\left(\varepsilon_{n}= \pm 1\right)$, is proved in [BKQ].

2.2. Criterion for algebraic genericity. From the topological point of view, a dense $G_{\delta}$ set in a Baire space is a very big set. But in an algebraic setting, a dense $G_{\delta}$ set may be very thin: in particular, there is no reason why it should contain an infinite-dimensional vector space. To name properties which hold on a big algebraic set, we say that a property on a topological vector space is algebraically generic if it holds for every non-zero vector in a dense subspace of $X$. If $T$ is a hypercyclic operator on a Banach space $X$ (meaning that the sequence of iterates $\left(T^{n}\right)_{n \geq 0}$ is hypercyclic), "being a hypercyclic vector" is a good example of an algebraically generic property: see [Bou] for the proof if $X$ is complex. One can ask if the divergence of Fourier series is algebraically generic. The following theorem says that the answer is yes. The method of proof could be easily adapted to other examples of divergent limiting processes. Before proceeding with the theorem, let us 
fix the notations: for $f \in L^{1}(\mathbb{T})$, we set

$$
\widehat{f}(n)=\frac{1}{2 \pi} \int_{\mathbb{T}} f(t) e^{-i n t} d t, \quad S_{N}(f, t)=\sum_{|n| \leq N} \widehat{f}(n) e^{i n t} .
$$

TheOREM 3. Let $X \subset L^{1}(\mathbb{T})$ be a Banach space and $E \subset \mathbb{T}$. Suppose that:

- The set of trigonometric polynomials is a dense subset of $X$ (for the topology of $X)$.

- For all $M>0$, there exists a trigonometric polynomial $Q$ such that $\|Q\|_{X}=1$ and $\sup _{N}\left|S_{N}(Q, t)\right|>M$ for all $t \in E$.

- $\left\|e^{i n t} f\right\|_{X}=\|f\|_{X}$ for all $n \in \mathbb{Z}$ and $f \in X$.

Then there exists a dense subspace $X_{0}$ of $X$ such that, for each $f \in X_{0} \backslash\{0\}$ and every $t \in E$, the Fourier series $\left(S_{n}(f, t)\right)_{n \geq 0}$ is unboundedly divergent.

Proof. Fix a sequence $\left(P_{l}\right)$ of trigonometric polynomials dense in $X$, and a sequence $\left(q_{l}\right)$ of integers such that $\operatorname{Sp}\left(P_{l}\right) \subset\left[-q_{l}, q_{l}\right]$ (here, $\operatorname{Sp}\left(P_{l}\right)$ denotes the spectrum of the polynomial $P_{l}$ ). We build by induction a sequence of "bad polynomials" with disjoint spectra. More precisely, we build, for $j \geq 1$ and $1 \leq k \leq j$, integers $n(j, k)$ and $m(j, k)$, and trigonometric polynomials $R(j, k)$ such that:

$$
\begin{aligned}
& \forall 1 \leq k<k^{\prime} \leq j, \\
& \qquad q_{j}<n(j, k) \leq m(j, k)<n\left(j, k^{\prime}\right) \leq m\left(j, k^{\prime}\right)<n(j+1,1) . \\
& \operatorname{Sp}(R(j, k)) \subset[n(j, k), m(j, k)] . \\
& \|R(j, k)\|_{X} \leq 1 / 2^{j+1} . \\
& \forall t \in E, \quad \sup _{n(j, k) \leq n \leq m(j, k)}\left|S_{n}(R(j, k), t)\right| \geq j .
\end{aligned}
$$

Indeed, for $j=1$, let $Q$ be a trigonometric polynomial such that:

- $\|Q\|_{X} \leq 1 / 2$.

- $\sup _{N}\left|S_{N}(Q, t)\right| \geq 1$ for all $t \in E$.

Then, if $\operatorname{Sp}(Q) \subset[-a, a]$, the polynomial $R(1,1)(t)=e^{i\left(a+q_{1}+1\right)} Q(t)$ works, with $n(1,1)=q_{1}+1$ and $m(1,1)=q_{1}+2 a+1$. Suppose now the construction is done till the rank $j-1$, and set $\beta=\max \left(q_{j}, m(j-1, j-1)\right)$. We fix a trigonometric polynomial $Q$ and an integer $a$ such that:

- $\|Q\|_{X} \leq 1 / 2^{j+1}$.

- $\sup _{N}\left|S_{N}(Q, t)\right| \geq j$ for all $t \in E$.

- $\operatorname{Sp}(Q) \subset[-a, a]$.

First we define

- $R(j, 1)(t)=e^{i(\beta+a+1) t} Q(t)$.

- $n(j, 1)=\beta+1$.

- $m(j, 1)=\beta+2 a+1$. 
Next, the sequences are defined by induction for $2 \leq k \leq j$, by setting

- $R(j, k)(t)=e^{i(m(j, k-1)+1) t} Q(t)$.

- $n(j, k)=m(j, k-1)+1$.

- $m(j, k)=m(j, k-1)+2 a+1$.

Clearly, conditions (2) to (5) are fulfilled. Finally, we set

$$
f_{k}=P_{k}+\sum_{j \geq k} R(j, k) .
$$

Observe that, for $k \neq k^{\prime}$,

$$
\operatorname{Sp}\left(\sum_{j \geq k} R(j, k)\right) \cap \operatorname{Sp}\left(\sum_{j \geq k^{\prime}} R\left(j, k^{\prime}\right)\right)=\emptyset .
$$

We claim that the vector space $X_{0}=\operatorname{span}\left(f_{k} ; k \geq 1\right)$ satisfies the requirements of Theorem 3. Indeed, on the one hand,

$$
\left\|f_{k}-P_{k}\right\|_{X} \leq \sum_{j \geq k}\|R(j, k)\| \leq 1 / 2^{k} .
$$

Therefore, $X_{0}$ is dense in $X$. On the other hand, pick any $f=a_{1} f_{1}+\cdots+$ $a_{N} f_{N}, a_{N} \neq 0$, in $X_{0} \backslash\{0\}$. By (5) and (6), for $j \geq N$ and $t \in E$, one has

$$
\sup _{n(j, N) \leq n \leq m(j, N)}\left|S_{n}(f, t)-S_{n(j, N)-1}(f, t)\right| \geq j\left|a_{N}\right| .
$$

The Fourier series $\left(S_{n}(f, t)\right)_{n \geq 0}$ is unboundedly divergent at each $t \in E$.

EXAMPLES. (1) If $X=L^{1}(\mathbb{T}), E=\mathbb{T}$, Kolmogorov's result gives exactly what is needed to apply Theorem 3 . Thus the algebraic genericity is proved for this example.

(2) The same proof works in the multi-dimensional case, and in particular for Fefferman's example: there exists a dense subspace $X_{0}$ of $L^{2}\left(\mathbb{T}^{2}\right)$ such that, for each $f \in X_{0} \backslash\{0\}$, and each $w \in \mathbb{T}^{2}$,

$$
\limsup _{|M|,|N| \rightarrow \infty}\left|S_{M, N}(f, w)\right|=\infty .
$$

(3) Let $X$ be $C(\mathbb{T})$, and $E \subset \mathbb{T}$ be a set of Lebesgue measure 0 . We can adapt the previous proof to produce a dense subspace $X_{0}$ of $C(\mathbb{T})$ such that, for each $f \in X_{0} \backslash\{0\}$, the Fourier series of $f$ at any point of $E$ is unboundedly divergent. Recall the following lemma from [KK]: given a finite union $F$ of intervals of $\mathbb{T}$, with Lebesgue measure $a, 0<a<1 / \pi$, there exists a trigonometric polynomial $Q$ with norm 1 in $C(\mathbb{T})$ such that

$$
\sup _{n \in \mathbb{N}}\left|S_{n}(Q, t)\right| \geq \frac{1}{\pi} \log \frac{1}{a \pi} \quad \text { when } t \in F .
$$


Since $E$ is negligible, we can find a sequence $\left(F_{j}\right)$ of sets such that $F_{j}$ is a finite union of intervals, its measure $a_{j}$ satisfies

$$
\frac{1}{\pi} \log \frac{1}{a_{j} \pi} \geq 2^{j+1} j
$$

and each point of $E$ belongs to infinitely many $F_{j}$ 's. The construction is the same, except that the polynomial $Q$ chosen at the $j$ th step is given by the lemma: it satisfies $\|Q\|_{C(\mathbb{T})} \leq 1 / 2^{j+1}$ and

$$
\sup _{n}\left|S_{n}(Q, t)\right| \geq j \quad \text { for each } t \in F_{j} .
$$

The rest of the proof is unchanged. Since each point of $E$ belongs to infinitely many $F_{j}$ 's, the Fourier series of each vector of $X_{0} \backslash\{0\}$ is unboundedly divergent on $E$.

(4) The proof cannot be extended to the case of the space $\mathcal{H}^{\infty}$ of Dirichlet series. More precisely, $\mathcal{H}^{\infty}$ is not separable (it contains $H^{\infty}(\mathbb{D})$ via the isometry $\left.\sum_{n \geq 0} a_{n} z^{n} \mapsto \sum_{n \geq 0} a_{n}\left(2^{n}\right)^{-s}\right)$, and the set of Dirichlet polynomials is not dense in $\mathcal{H}^{\infty}$. So the proof just gives an infinite-dimensional subspace $X_{0}$ of $\mathcal{H}^{\infty}$, not necessarily dense, such that, for each $f=\sum_{n \geq 1} a_{n} n^{-s} \in X_{0} \backslash\{0\}$, the series $\sum_{n \geq 1} a_{n} n^{i t}$ diverges for each $t \in \mathbb{R}$.

\section{Universal series}

3.1. Topological and algebraic genericity. The first result of this section is a general result which states clearly, for universal series, the following well known fact (see [Gre]): "Universality is a generic phenomenon in analysis".

TheOREm 4. Let $X$ be a separable metrizable vector space, and $Y$ be a subset of $\mathbb{C}^{\mathbb{N}}$ such that:

(a) $Y$ is a Baire topological space.

(b) The projection on each component is continuous.

(c) If $a=\left(a_{n}\right), b=\left(b_{n}\right) \in Y$, and $N \geq 0$, then

$$
c(N)=\left(b_{0}, \ldots, b_{N}, a_{N+1}, a_{N+2}, \ldots\right)
$$

belongs to $Y$ and $c(N) \rightarrow b$ in $Y$ as $N \rightarrow \infty$.

Finally, let $\left(x_{n}\right)$ be a sequence of vectors in $X$. Then the following are equivalent:

(i) For at least one $a \in Y$, the series $\sum a_{n} x_{n}$ is universal.

(ii) For quasi-all $a \in Y$, the series $\sum a_{n} x_{n}$ is universal. 
Proof. Fix a countable basis $\left(U_{l}\right)$ of open sets in $X$, and set

$$
G(l)=\left\{a \in Y ; \exists N \geq 0 \text { such that } \sum_{n=0}^{N} a_{n} x_{n} \in U_{l}\right\} .
$$

By condition (b), $G(l)$ is open. Moreover, $G(l)$ is dense: indeed, consider $b \in Y$, an open set $U$ containing $b$, and $a \in Y$ so that $\sum a_{n} x_{n}$ is universal. By condition (c), there exists $N_{0} \in \mathbb{N}$ such that

$$
c=\left(b_{0}, \ldots, b_{N_{0}}, a_{N_{0}+1}, \ldots\right) \in U .
$$

Since $\sum a_{n} x_{n}$ is universal, there exists $N_{1} \geq N_{0}$ such that

$$
\sum_{n=0}^{N_{1}} a_{n} x_{n} \in U_{l}+\sum_{n=0}^{N_{0}} a_{n} x_{n}-\sum_{n=0}^{N_{0}} b_{n} x_{n} .
$$

Therefore,

$$
\sum_{n=0}^{N_{1}} c_{n} x_{n} \in U_{l},
$$

which means that $c \in G(l) \cap U$. Clearly, each sequence in $\bigcap_{l \geq 1} G(l)$ gives a universal series.

The hypotheses of Theorem 4 are natural and very permissive. For instance, $Y$ could be $c_{0}, \ell^{p}, \mathbb{C}^{\mathbb{N}}$ with the topology of convergence of the coefficients, or the set of all choices of signs with the same topology (see below). In case $Y$ is a topological vector space which contains the set of finitely supported sequences as a dense subspace, Theorem 4 is a consequence of already known results on hypercyclic sequences. Indeed, as announced in the introduction, the existence of a universal series is equivalent to the hypercyclicity of the sequence of operators $\left(T_{N}\right)$, with $T_{N}: Y \rightarrow X$ defined by $T_{N}(a)=\sum_{n=0}^{N} a_{n} x_{n}$. Since $\left(T_{N}\right)$ converges pointwise on the dense set of finitely supported sequences, Proposition 6 of [Gre] ensures that the set of hypercyclic vectors (equivalently, the set of universal series) is either empty or residual.

On the other hand, we obtain new examples of topological genericity for sets which are not vector spaces.

COROLlary 1. Let $Y$ be $\mathbb{T}^{\mathbb{N}}$, equipped with the natural topology defined by convergence of the coefficients for each index $n$. Let $H$ be a separable Hilbert space, and $\left(x_{n}\right)$ be a sequence in $H$ satisfying:

(a) $\sum_{n \geq 0}\left\|x_{n}\right\|^{2}<\infty$. 
(b) $\sum_{n \geq 0}\left|\left\langle x_{n}, x\right\rangle\right|=\infty$ for all $x \in H \backslash\{0\}$.

Then, for quasi-all $a \in Y$, the series $\sum_{n \geq 0} a_{n} x_{n}$ is universal.

Proof. By [La, p. 208], the set of convergent series $\left\{\sum_{n \geq 0} a_{n} x_{n} ;\left|a_{n}\right|\right.$ $=1\}$ is dense in $H$. In particular, if we fix $n_{0} \geq 0$, the set of "polynomials"

$$
\left\{\sum_{n=n_{0}}^{N} a_{n} x_{n} ; N \geq n_{0},\left|a_{n}\right|=1\right\}
$$

is dense in $H$. We deduce the existence of a universal series by the following argument: let $\left(z_{k}\right)_{k \geq 1}$ be a dense sequence in $H$. By induction, one exhibits a sequence $\left(a_{j}\right)_{j \geq 0}$ of unimodular complex numbers and an increasing sequence $\left(n_{k}\right)_{k \geq 1}$ of integers such that

$$
\left\|z_{k}-\sum_{n=0}^{n_{k}} a_{n} x_{n}\right\| \leq \frac{1}{k}
$$

- For $k=1$, this is obvious.

- Suppose that $n_{1}<\cdots<n_{k}$ have already been chosen, satisfying (7) at step $k$. There exist $n_{k+1}>n_{k}$ and $a_{n_{k}+1}, \ldots, a_{n_{k+1}}$, with $\left|a_{j}\right|=1$, such that

$$
\left\|\left(z_{k+1}-\sum_{n=0}^{n_{k}} a_{n} x_{n}\right)-\sum_{n=n_{k}+1}^{n_{k+1}} a_{n} x_{n}\right\| \leq \frac{1}{k+1} .
$$

It follows from (7) that the series $\sum a_{n} x_{n}$ is universal, and Theorem 4 gives the topological genericity.

REMARK. $\mathbb{T}^{\mathbb{N}}$ could also be viewed as a probability space, equipped with the product of the Haar measure on each factor. If $\left(x_{n}\right)$ satisfies the assumptions of Corollary 1, Kolmogorov's Theorem asserts that, for almost all $\left(a_{n}\right) \in \mathbb{T}^{\mathbb{N}}$, the series $\sum_{n=0}^{\infty} a_{n} x_{n}$ is convergent. This is in strong contrast with the quasi-sure behavior of such series, since for quasi-all $\left(a_{n}\right) \in \mathbb{T}^{\mathbb{N}}$, the series $\sum_{n=0}^{\infty} a_{n} x_{n}$ is divergent, in the worst possible sense, because it is universal. As usual, the Baire approach emphasizes divergence, while the probabilistic one favors convergence.

Theorem 3 and Theorem 2 of [Be] suggest studying the algebraic genericity of universal series:

TheOREM 5. Let $X$ be a metrizable topological vector space, and $Y$ be a subspace of $\mathbb{C}^{\mathbb{N}}$ such that:

(a) $Y$ is a Baire topological space.

(b) The projection on each component is continuous. 
(c) If $a=\left(a_{n}\right) \in Y$ and $N \geq 0$, then

$$
b(N)=\left(a_{0}, \ldots, a_{N}, 0, \ldots\right)
$$

belongs to $Y$ and $b(N) \rightarrow a$ as $N \rightarrow \infty$.

Finally, let $\left(x_{n}\right)$ be a sequence of vectors in $X$. Then the following are equivalent:

(i) For at least one $a \in Y$, the series $\sum a_{n} x_{n}$ is universal.

(ii) For quasi-all $a \in Y$, the series $\sum a_{n} x_{n}$ is universal.

(iii) There exists a dense subspace $Y_{0}$ of $Y$ such that, for all $a \in Y_{0} \backslash\{0\}$, $\sum a_{n} x_{n}$ is universal.

Proof. It suffices to prove (i) $\Rightarrow\left(\right.$ iii). Recall ([Be]) that a sequence $\left(T_{N}\right)$ of operators from $Y$ to $X$ is densely hypercyclic if it is hypercyclic and the set of its hypercyclic vectors is dense in $Y$. It is called densely hereditarily hypercyclic if, for each increasing sequence $\left(N_{k}\right)_{k \geq 0}$ of integers, the sequence of operators $\left(T_{N_{k}}\right)_{k \geq 0}$ is densely hypercyclic. The Theorem of Bernal-González mentioned in the introduction asserts that, if $X$ and $Y$ are two separable metrizable vector spaces, and if $T_{N}: Y \rightarrow X$ is a densely hereditarily hypercyclic sequence of operators, then the set of its hypercyclic vectors contains a dense subspace of $Y$ (without 0 ).

Thus, it suffices to prove that the sequence $\left(T_{N}\right)_{N \geq 0}$ of operators with

$$
T_{N}: Y \rightarrow X, \quad\left(a_{n}\right) \mapsto \sum_{n=0}^{N} a_{n} x_{n},
$$

is densely hereditarily hypercyclic. Fix an increasing sequence $\left(N_{k}\right)$ of integers, and let $a \in Y$ be such that $\sum a_{n} x_{n}$ is universal. Moreover, fix an open subset $U$ of $Y$, and an open subset $V$ of $X$. For a sequence $b$ in $U$, by condition (c), there exists $m \geq 0$ such that, for each $l \geq m$,

$$
\left(b_{0}, \ldots, b_{m}, a_{m+1}, \ldots, a_{l}, 0, \ldots\right) \in U \text {. }
$$

Since $\sum a_{n} x_{n}$ is universal, there exists $u \geq m$ such that

$$
\sum_{n=0}^{u} a_{n} x_{n} \in V+\sum_{n=0}^{m} a_{n} x_{n}-\sum_{n=0}^{m} b_{n} x_{n} .
$$

Set $c=\left(b_{0}, \ldots, b_{m}, a_{m+1}, \ldots, a_{u}, 0, \ldots\right)$. Summarizing, if $k$ is chosen so that $N_{k}>u$, one has

$$
c \in U, \quad T_{N_{k}}(c) \in V .
$$

By the hypercyclicity criterion (see [Gre, Thm 1]), the sequence $\left(T_{N_{k}}\right)_{k \geq 0}$ is densely hypercyclic.

Example. J.-P. Kahane and V. Nestoridis have proved in $[\mathrm{KN}]$ the existence of a sequence $a$ in $c_{0}(\mathbb{Z})$ such that the trigonometric series $\sum_{n=-\infty}^{\infty} a_{n} e^{i n t}$ is universal in the sense of Men'shov: every measurable 
function on $\mathbb{T}$ can be approximated by some sequences of partial sums $\sum_{n=-N}^{N} a_{n} e^{i n t}$ almost everywhere on $\mathbb{T}$. Actually, they prove that quasi-all sequences of $c_{0}(\mathbb{Z})$ give a universal series in the sense of Men'shov. The last theorem shows that this property also holds for a dense subspace of $c_{0}(\mathbb{Z})$, without 0 .

3.2. Universality and Dirichlet series. Our aim in this section is to extend Nestoridis' universal theorem to the case of Dirichlet series. Let $\mathcal{D}_{\mathrm{a}}\left(\mathbb{C}_{+}\right)$ denote the space of all Dirichlet series which are absolutely convergent in the right half-plane $\mathbb{C}_{+}$. It is a Fréchet space, endowed with the family of seminorms

$$
\left\|\sum_{n=1}^{\infty} a_{n} n^{-s}\right\|_{\sigma}=\sum_{n=1}^{\infty}\left|a_{n}\right| n^{-\sigma}, \quad \sigma>0 .
$$

Our main tool for approximation by Dirichlet series is the following lemma from Bagchi [Bag]:

Lemma 1. Let $g$ be an analytic function in the strip $\{s \in \mathbb{C} ; 1 / 2<$ $\Re(s)<1\}, K$ be a compact set contained in the same strip, $n_{0}$ be an integer and $\varepsilon>0$. Then there exist $n_{1}>n_{0}$ and complex numbers $\left(b_{j}\right)_{j=n_{0}+1}^{n_{1}}$, with $\left|b_{j}\right| \leq 1$, such that, for any $s \in K$,

$$
\left|g(s)-\sum_{j=n_{0}+1}^{n_{1}} b_{j} j^{-s}\right|<\varepsilon .
$$

We will deduce from this lemma a version of Mergelyan's Theorem for Dirichlet series, but first of all we need to introduce a definition:

Definition 1. A compact set $K \subset \mathbb{C}$ is said to be admissible for Dirichlet series if $\mathbb{C} \backslash K$ is connected, and if it can be written as $K=K_{1} \cup \cdots \cup K_{d}$, each $K_{i}$ being contained in a strip $S_{i}=\left\{s \in \mathbb{C} ; a_{i} \leq \Re(s) \leq b_{i}\right\}$, with $b_{i}-a_{i}<1 / 2$, the strips $S_{i}$ being disjoint.

\section{Let $\mathbb{C}_{-}=\{s \in \mathbb{C} ; \Re(s)<0\}$.}

Lemma 2. Let $K \subset \overline{\mathbb{C}}_{-}$be a compact set admissible for Dirichlet series, $f$ be in $\mathcal{D}_{\mathrm{a}}\left(\mathbb{C}_{+}\right), g$ be a continuous function on $K$, analytic in $\stackrel{\circ}{K}$, and $\sigma, \varepsilon$ two positive numbers. Then there exists $h$ in $\mathcal{D}_{a}\left(\mathbb{C}_{+}\right)$such that

$$
\|h-g\|_{C(K)}<\varepsilon, \quad\|h-f\|_{\sigma}<\varepsilon .
$$

Proof. By Mergelyan's Theorem, we may suppose that $g$ is an entire function (and even that $g$ is a polynomial). Write $K=K_{1} \cup \cdots \cup K_{d}$ with $K_{i} \subset\left\{s \in \mathbb{C} ; a_{i} \leq \Re(s) \leq b_{i}\right\}$, and assume $0 \geq b_{1} \geq a_{1}>b_{2} \geq \cdots>b_{d} \geq$ $a_{d}, b_{i}-a_{i}<1 / 2$. We first approximate simultaneously $f(s)=\sum_{j=1}^{\infty} a_{j} j^{-s}$ 
and $g$ on $K_{1}$. We fix $\sigma_{1} \in \mathbb{R}$ such that $K_{1}+\sigma_{1} \subset\{s \in \mathbb{C} ; 1 / 2<\Re(s)<1\}$, and $\sigma+\sigma_{1}>1$. Consider $n_{1} \in \mathbb{N}$ such that

$$
\sum_{j \geq n_{1}+1} \frac{1}{j^{\sigma_{1}+\sigma}}<\frac{\varepsilon}{2}, \quad\left\|f-\sum_{j=1}^{n_{1}} a_{j} j^{-s}\right\|_{\sigma}<\frac{\varepsilon}{2} .
$$

By Lemma 1, there exist $m_{1}>n_{1}$ and complex numbers $\left(b_{j}^{(1)}\right)_{n=n_{1}+1}^{m_{1}}$ with $\left|b_{j}^{(1)}\right| \leq 1$ such that

$$
\forall s \in K_{1}+\sigma_{1}, \quad\left|\left(g\left(s-\sigma_{1}\right)-\sum_{j=1}^{n_{1}} a_{j} j^{-s+\sigma_{1}}\right)-\sum_{j=n_{1}+1}^{m_{1}} b_{j}^{(1)} j^{-s}\right|<\frac{\varepsilon}{2} .
$$

We next approximate inductively $g$ on each $K_{i}, i \geq 2$, without affecting the previous approximations. Let $\sigma_{i} \in \mathbb{R}$ be such that

$$
1 / 2<a_{i}+\sigma_{i} \leq b_{i}+\sigma_{i}<1 \text { and } 1<a_{i-1}+\sigma_{i} .
$$

We define $\alpha_{i}=a_{i-1}+\sigma_{i}>1$. There exists $n_{i}>m_{i-1}$ such that

$$
\sum_{j \geq n_{i}+1} \frac{1}{j^{\alpha_{i}}}<\frac{\varepsilon}{2}
$$

By Lemma 1 , there exist $m_{i}>n_{i}$ and complex numbers $\left(b_{j}^{(i)}\right)_{j=n_{i}+1}^{m_{i}}$ with $\left|b_{j}^{(i)}\right| \leq 1$ such that, for any $s \in K_{i}+\sigma_{i}$,

$$
\left|\left(g\left(s-\sigma_{i}\right)-\sum_{j=1}^{n_{1}} a_{j} j^{-s+\sigma_{i}}-\sum_{l=1}^{i-1} \sum_{j=n_{l}+1}^{m_{l}} b_{j}^{(l)} j^{-s+\sigma_{i}-\sigma_{l}}\right)-\sum_{j=n_{i}+1}^{m_{i}} b_{j}^{(i)} j^{-s}\right|<\frac{\varepsilon}{2} .
$$

We finally set

$$
h(s)=\sum_{j=1}^{n_{1}} a_{j} j^{-s}+\sum_{l=1}^{d} \sum_{j=n_{l}+1}^{m_{l}} b_{j}^{(l)} j^{-\sigma_{l}} j^{-s},
$$

and we claim that $h$ satisfies the conclusions of Lemma 2. Indeed,

$$
\begin{aligned}
\|h-f\|_{\sigma} & \leq \frac{\varepsilon}{2}+\sum_{l=1}^{d} \sum_{j=n_{l}+1}^{m_{l}} \frac{\left|b_{j}^{(l)}\right|}{j^{\sigma+\sigma_{l}}} \\
& \leq \frac{\varepsilon}{2}+\sum_{j=n_{1}+1}^{\infty} \frac{1}{j^{\sigma_{1}+\sigma}} \leq \varepsilon .
\end{aligned}
$$

Now, take any $i$ in $\{1, \ldots, d\}$, and $s \in K_{i}$. One has 


$$
\begin{aligned}
& |h(s)-g(s)|=\left|h\left(s+\sigma_{i}-\sigma_{i}\right)-g\left(s+\sigma_{i}-\sigma_{i}\right)\right| \\
& \leq\left|\left(\left(s+\sigma_{i}\right)-\sigma_{i}\right)-\sum_{1}^{n_{1}} a_{j} j^{-\left(s+\sigma_{i}\right)+\sigma_{i}}-\sum_{l=1}^{i} \sum_{j=n_{l}+1}^{n_{l+1}} b_{j}^{(l)} j^{-\left(s+\sigma_{i}\right)+\sigma_{i}-\sigma_{l} \mid}\right| \\
& \quad+\sum_{l=i+1}^{d} \sum_{j=n_{l}+1}^{m_{l}} j^{-\left(\sigma_{l}+a_{i}\right)} \\
& \leq \frac{\varepsilon}{2}+\sum_{j \geq n_{i}+1} \frac{1}{j^{\alpha_{i}}} \leq \varepsilon .
\end{aligned}
$$

We are now ready to prove the existence of universal Dirichlet series "à la Nestoridis":

TheOREM 6. There exists a Dirichlet series $S=\sum_{n \geq 1} a_{n} n^{-s}$ in $\mathcal{D}_{\mathrm{a}}\left(\mathbb{C}_{+}\right)$ which is universal in the following sense: For each compact set $K \subset \mathbb{C}$ such that $K \subset\{s \in \mathbb{C} ;-1 / 2<\Re(s) \leq 0\}$ and $\mathbb{C} \backslash K$ is connected, and for any function $g$ continuous on $K$ and analytic in the interior of $K$, there exists a sequence of partial sums of $S$ which converges to $g$ uniformly on $K$. Moreover, this property is topologically and algebraically generic in $\mathcal{D}_{\mathrm{a}}\left(\mathbb{C}_{+}\right)$.

Proof. Let $Y$ be the Fréchet space $\mathcal{D}_{\mathrm{a}}\left(\mathbb{C}_{+}\right)$and $X$ be the space of all functions which are continuous on $\{s \in \mathbb{C} ;-1 / 2<\Re(s) \leq 0\}=: \Omega_{-1 / 2,0}$ and analytic in the interior of $\Omega_{-1 / 2,0}$, endowed with the topology of uniform convergence on compact subsets of $\Omega_{-1 / 2,0}$. We also introduce the sequence of operators

$$
T_{N}: Y \rightarrow X, \quad \sum_{n=1}^{\infty} a_{n} n^{-s} \mapsto \sum_{n=1}^{N} a_{n} n^{-s},
$$

and prove that this sequence is hereditarily hypercyclic. To this end, fix an increasing sequence $\left(N_{k}\right)_{k \geq 0}$ of integers, $f \in Y, g \in X, \sigma>0$, a compact subset $K$ of $\{s \in \mathbb{C} ;-1 / 2<\Re(s) \leq 0\}$ and $\varepsilon>0$. Then $K$ is included in a rectangle $K_{1} \subset\{s \in \mathbb{C} ;-1 / 2<\Re(s) \leq 0\}$. By Lemma 2, there exists a Dirichlet polynomial $P(s)=\sum_{n=1}^{N} a_{n} n^{-s}$ such that

$$
\|f-P\|_{\sigma}<\varepsilon, \quad\|g-P\|_{C\left(K_{1}\right)}<\varepsilon \text {. }
$$

For $k$ so that $N_{k} \geq N$, one has

$$
\|f-P\|_{\sigma}<\varepsilon, \quad\left\|g-T_{N_{k}} P\right\|_{C(K)}<\varepsilon .
$$

The hypercyclicity criterion ensures that $\left(T_{N_{k}}\right)_{k \geq 0}$ is densely hypercyclic, and the set of hypercyclic vectors for $\left(T_{N_{k}}\right)$ contains a residual set. We deduce that the set of hypercyclic vectors for $\left(T_{N}\right)$ is topologically and algebraically generic. Theorem 6 follows, since by Mergelyan's Theorem any hypercyclic vector for $\left(T_{N}\right)$ is clearly a universal Dirichlet series. 
Remarks. (1) It is possible to prove the same assertion with $\mathcal{D}_{\mathrm{a}}\left(\mathbb{C}_{+}\right)$ replaced by $\mathcal{D}_{\mathrm{u}}\left(\mathbb{C}_{+}\right)$, the space of Dirichlet series which converge uniformly on each half-plane $\mathbb{C}_{\varepsilon}=\{s \in \mathbb{C} ; \Re(s)>\varepsilon\}, \varepsilon>0$, endowed with the topology of uniform convergence on these half-planes. The proof is the same.

(2) A slight modification of the proof (for instance, a direct proof instead of a topological one) shows that we can build a universal Dirichlet series $\sum_{j>1} a_{j} j^{-s}$ satisfying $a_{j}=o\left(j^{-r}\right)$ for any $r<1$.

(3) Our method of proof differs from that of Nestoridis in that we use the hypercyclicity criterion instead of reproving it by applying Baire's Theorem. This method can also be applied for the Nestoridis Theorem, in order to obtain the algebraic genericity.

(4) As in Section 3 of [Ne], we can obtain the existence of a Dirichlet series universal in the sense of Men'shov. More precisely, if $S=\sum_{n>1} a_{n} n^{-s}$ is a universal Dirichlet series in the sense of Nestoridis, and if $h, g: \mathbb{R} \rightarrow[-\infty, \infty]$ are two measurable functions, then there exists a subsequence $S_{k_{m}}$ of the partial sums of $S$ such that

$$
\Re\left(S_{k_{m}}(i t)\right) \rightarrow g(t) \quad \text { and } \quad \Im\left(S_{k_{m}}(i t)\right) \rightarrow h(t)
$$

as $m \rightarrow \infty$, almost everywhere on $\mathbb{R}$. The universality in the sense of Men'shov is topologically and algebraically generic in $\mathcal{D}_{\mathrm{a}}\left(\mathbb{C}_{+}\right)$.

(5) Actually, our method of proof gives a stronger conclusion than stated in Theorem 6 . Let $K_{1}, K_{2}, \ldots$ be a sequence of compact subsets of $\overline{\mathbb{C}}_{-}$such that each compact set in $\overline{\mathbb{C}}_{-}$admissible for Dirichlet series is contained in at least one $K_{i}$. We can take, for instance, all finite unions of rectangles whose vertices have coordinates in $\overline{\mathbb{Q}}_{-}+i \mathbb{Q}$ and which are admissible for Dirichlet series. Denote by $X_{i}$ the Banach space of functions continuous on $K_{i}$ and analytic in the interior of $K_{i}$. As in the proof of Theorem 6 , the sequence of operators

$$
T_{N}: \mathcal{D}_{a}\left(\mathbb{C}_{+}\right) \rightarrow X_{i}, \quad \sum_{n=1}^{\infty} a_{n} n^{-s} \mapsto \sum_{n=1}^{N} a_{n} n^{-s},
$$

is hereditarily hypercyclic. Denote by $H_{i}$ the set of its hypercyclic vectors, which is a dense $G_{\delta}$ subset of $\mathcal{D}_{\mathrm{a}}\left(\mathbb{C}_{+}\right)$. Let $S$ be in $\bigcap_{i \geq 1} H_{i} \neq \emptyset$. Then $S$ is universal in the following sense: For each compact set $K \subset \overline{\mathbb{C}}_{-}$admissible for Dirichlet series, and any function $g$ continuous on $K$ and analytic in the interior of $K$, there exists a sequence of partial sums of $S$ which converges to $g$ uniformly.

(6) We do not know if Theorem 6 remains true for arbitrary compact subsets of $\overline{\mathbb{C}}_{-}$whose complement is connected.

4. Common algebraic genericity. Let $I$ be a set, and $\left(T_{n, \lambda}\right)_{n \geq 1, \lambda \in I}$ be a family of operators on a separable normed space $X$. For convenience 
of notation, for each $\lambda \in I, T_{0, \lambda}$ will always be taken equal to $\operatorname{Id}_{X}$. Suppose that, for each $\lambda \in I$, the sequence $\left(T_{n, \lambda}\right)_{n \in \mathbb{N}}$ is hypercyclic, and denote by $\mathrm{HC}\left(T_{., \lambda}\right)$ the set of its hypercyclic vectors:

$$
\mathrm{HC}\left(\left(T_{., \lambda}\right)\right)=\left\{x \in X ;\left(T_{n, \lambda} x\right)_{n \geq 0} \text { is dense in } X\right\} .
$$

It is natural to study the structure of $\bigcap_{\lambda \in I} \mathrm{HC}\left(T_{., \lambda}\right)$. Is $\bigcap_{\lambda \in I} \mathrm{HC}\left(T_{., \lambda}\right)$ nonempty? Does it contain a dense $G_{\delta}$ set? Does it contain a dense subspace? The first positive answer was given by E. Abakumov and J. Gordon who proved that $\bigcap_{\lambda>1} \operatorname{HC}(\lambda B) \neq \emptyset$, where $B$ is the backward shift on $\ell^{2}$.

To proceed further, it is convenient to introduce the following

Definition 2. Let $\left(T_{n, \lambda}\right)_{n \in \mathbb{N}, \lambda \in I}$ be a family of sequences of operators on $X, I$ being an interval of the real line. We say that the family satisfies (CHC), the common hypercyclic criterion, provided there exists a family of operators $\left(S_{n, \lambda}\right)_{n \in \mathbb{N}, \lambda \in I}$ with $T_{n, \lambda} \circ S_{n, \lambda}=\operatorname{Id}_{X}$, and a sequence $\left(x_{j}\right)$ dense in $X$ such that:

(1) Given a compact set $K \subset I$ and an integer $n_{0}$, there exists a sequence $\left(c_{k}\right)$ of positive numbers with:

(a) $\sum c_{k}<\infty$

(b) $\left\|T_{n+k, \lambda} S_{n, \alpha}\left(x_{j}\right)\right\| \leq c_{k}$ for any $n, k \geq 0$, and $\lambda, \alpha \in K, \lambda \geq \alpha$.

(c) $\left\|T_{n, \lambda} S_{n+k, \alpha}\left(x_{j}\right)\right\| \leq c_{k}$ for any $n, k \geq 0$, and $\lambda, \alpha \in K, \lambda \leq \alpha$.

(d) $\left\|T_{k, \lambda} S_{n_{0}, \alpha}\left(x_{j}\right)\right\| \stackrel{k \rightarrow \infty}{\longrightarrow} 0$ for $\lambda, \alpha \in K$, uniformly in $\lambda$.

(e) $\left\|T_{n, \lambda} S_{n+k, \alpha}\left(x_{j}\right)\right\| \leq c_{k}$ for any $k \geq 0, n \leq n_{0}$ and $\lambda, \alpha \in K$.

(2) Given $\varepsilon>0$ and a compact set $K \subset I$, there exists $\delta=\delta\left(\varepsilon, x_{j}, K\right)$ such that

$$
|\lambda-\alpha| \leq \delta / n \Rightarrow\left\|T_{n, \lambda} S_{n, \alpha}\left(x_{j}\right)-x_{j}\right\| \leq \varepsilon .
$$

In [CS], it is proved that if $\left(T_{n, \lambda}\right)$ satisfies $(\mathrm{CHC})$, then $\bigcap_{\lambda \in I} \mathrm{HC}\left(T_{., \lambda}\right)$ contains a residual set (actually, this is done under slightly weaker assumptions). In [Ba2], it is proved that, if $\left(T_{n, \lambda}\right)$ satisfies $(\mathrm{CHC})$, if each $T_{n, \lambda}$ can be written, for $\lambda$ fixed, as a power of a single operator, $T_{n, \lambda}=T_{\lambda}^{n}$, if $X$ is a Banach space and if there exists a closed infinite-dimensional subspace $M$ of $X$ such that, for every vector $x$ in $M$ and every $\lambda \in I,\left\|T_{\lambda}^{n} x\right\| \rightarrow 0$ as $n \rightarrow \infty$, then there exists a closed infinite-dimensional subspace $X_{0} \subset X$ such that $X_{0} \backslash\{0\} \subset \bigcap_{\lambda \in I} \mathrm{HC}\left(T_{., \lambda}\right)$.

Here, we prove the algebraic genericity.

THEOREM 7. If $X$ is a Banach space, and if $\left(T_{n, \lambda}\right)$ is a family of operators acting on $X$ and satisfying $(\mathrm{CHC})$, then there exists a dense subspace $X_{0} \subset X$ such that

$$
X_{0} \backslash\{0\} \subset \bigcap_{\lambda \in I} \operatorname{HC}\left(T_{., \lambda}\right) .
$$


Proof. In [CS], the existence of a common hypercyclic vector is shown by using a Baire category argument. Here, we need more than a common hypercyclic vector: we have to control how it approaches zero and any vector. Baire's method is not accurate enough, and our proof is constructive. First, fix an increasing sequence of compact sets $K_{k} \subset I$ such that $I=\bigcup_{k>1} K_{k}$. For $l \geq k \geq 1$, we build vectors $z_{k}(l)$ and blocks of integers $\Gamma_{k, l}$ such that:

- $z_{l}(l)$ is close to $x_{l}$ :

$$
\left\|z_{l}(l)-x_{l}\right\|<1 / 2^{l}
$$

- $\forall l \geq 1, \forall k \leq l, \forall \lambda \in K_{l}, \exists n=n(l, k, \lambda) \in \Gamma_{k, l}$ such that

$$
\begin{gathered}
\left\|T_{n, \lambda} z_{k}(l)-x_{l}\right\|<1 / 2^{l}, \\
\sum_{j<l}\left\|T_{n, \lambda} z_{k}(j)\right\|<1 / 2^{l}, \\
\forall m<k, \quad \sum_{j \leq l}\left\|T_{n, \lambda} z_{m}(j)\right\|<1 / 2^{l} .
\end{gathered}
$$

- $\forall l \geq 1, \forall m<l, \forall \lambda \in K_{l}, \forall n \leq \max _{1 \leq j<l, 1 \leq r \leq j} \Gamma_{r, j}$

$$
\left\|T_{n, \lambda} z_{m}(l)\right\|<\frac{1}{2^{l+1}} .
$$

One easily deduces Theorem 7 from the existence of such sequences. Indeed, set

$$
z_{k}=\sum_{l \geq k} z_{k}(l)
$$

By (8) and (12), applied to $n=0, z_{k}$ is well defined and the sequence $\left(z_{k}\right)_{k \geq 1}$ is dense in $X$. We define $X_{0}=\operatorname{span}\left(z_{k} ; k \geq 1\right)$, and we claim that every vector $z=a_{1} z_{1}+\cdots+a_{k} z_{k} \in X_{0}$ with $a_{k}=1$ is hypercyclic for the family $\left(T_{n, \lambda}\right)_{n \geq 0}$, for any $\lambda \in I$. Indeed, fix $\lambda \in I$ and $l_{0}>k$ such that $l \geq l_{0} \Rightarrow \lambda \in \bar{K}_{l}$. To any $l \geq l_{0}$, one can associate $n=n(l, k, \lambda) \in \Gamma_{k, l}$ such that (9)-(11) are simultaneously true. On the other hand,

$$
\begin{aligned}
\left\|T_{n, \lambda} z-x_{l}\right\| \leq & \left\|T_{n, \lambda} z_{k}(l)-x_{l}\right\| \\
& +\sum_{j=k}^{l-1}\left\|T_{n, \lambda} z_{k}(j)\right\|+\sum_{j=l+1}^{\infty}\left\|T_{n, \lambda} z_{k}(j)\right\| \\
& +\sum_{m=1}^{k-1}\left|a_{m}\right|\left(\sum_{j=m}^{l}\left\|T_{n, \lambda} z_{m}(j)\right\|+\sum_{j=l+1}^{\infty}\left\|T_{n, \lambda} z_{m}(j)\right\|\right) .
\end{aligned}
$$

Now, the following inequalities hold:

- $\left\|T_{n, \lambda} z_{k}(l)-x_{l}\right\|<1 / 2^{l}$, by $(9)$. 
- $\sum_{j=k}^{l-1}\left\|T_{n, \lambda} z_{k}(j)\right\|<1 / 2^{l}$, by $(10)$.

- $\sum_{j=m}^{l}\left\|T_{n, \lambda} z_{m}(j)\right\|<1 / 2^{l}$ for $m<k$, by (11).

- $\sum_{j=l+1}^{\infty}\left\|T_{n, \lambda} z_{m}(j)\right\|<1 / 2^{l}$ for $m \leq k$, by $(12)$.

Finally, for any $\varepsilon>0$, we can choose $l_{0}$ large enough to get

$$
l \geq l_{0} \Rightarrow\left\|T_{n, \lambda} z-x_{l}\right\|<\varepsilon .
$$

Since the sequence $\left(x_{l}\right)_{l \geq l_{0}}$ remains dense, $z \in \bigcap_{\lambda \in I} \operatorname{HC}\left(T_{., \lambda}\right)$.

It remains to build $z_{k}(l)$ and $\Gamma_{k, l}$, for $1 \leq k \leq l$. We proceed by induction on $l$.

STEP 1. It is enough to take $z_{1}(1)=x_{1}$, and $\Gamma_{1,1}=\{0\}$.

STEP $l$. We assume that vectors $z_{r}(j)$ and blocks of integers $\Gamma_{r, j}$ have been built in the previous steps, with the property that each $z_{r}(j)$ is a (finite) linear combination of $S_{n, \alpha}\left(x_{j}\right)$, with $n \in \Gamma_{r, j}$. Set $n_{0}=\max _{1 \leq j<l, 1 \leq r \leq j} \Gamma_{r, j}$. Let $\left(c_{n}\right)$ be the sequence introduced in Definition 2, for the parameters $x_{l}$, $K_{l}=[a, b]$ and $n_{0}$. Observe that, by $(\mathrm{CHC})(1)(\mathrm{d})$, since $z_{1}(j)$ is a linear combination of $S_{n, \alpha}\left(x_{j}\right)$, we have

$$
\forall \lambda \in K_{l}, \forall j \leq l-1, \quad\left\|T_{n, \lambda} z_{1}(j)\right\| \rightarrow 0
$$

as $n \rightarrow \infty$, uniformly in $\lambda \in K_{l}$. Therefore it is possible to find an integer $u>n_{0}$ such that

$$
\forall n \geq u, \forall \lambda \in K_{l}, \quad \sum_{j<l}\left\|T_{n, \lambda} z_{1}(j)\right\|<1 / 2^{l}
$$

and

$$
\sum_{v \geq u-n_{0}} c_{v}<1 / 2^{l+2}
$$

We fix $\delta=\delta\left(1 / 2^{l+1}, x_{l}, K_{l}\right)$ and consider a subdivision $a=\alpha_{0}<\alpha_{1}<\cdots<$ $\alpha_{m}=b$ of $K_{l}$ with

$$
\alpha_{i+1}-\alpha_{i} \leq \frac{\delta}{u(i+1)}
$$

This is always possible, for instance by choosing $\alpha_{0}=a, \alpha_{1}=a+\delta / u$, $\alpha_{2}=\alpha_{1}+\delta / 2 u, \ldots$ Finally, we set

$$
z_{1}(l)=S_{u, \alpha_{0}}\left(x_{l}\right)+\cdots+S_{m u, \alpha_{m-1}}\left(x_{l}\right), \quad \Gamma_{1, l}=\{u, 2 u, \ldots, m u\} .
$$


For any $\lambda \in K_{l}$, take $j \in\{0, \ldots, m-1\}$ such that $\alpha_{j} \leq \lambda \leq \alpha_{j+1}$. Then

$$
\begin{aligned}
\left\|T_{(j+1) u, \lambda} z_{1}(l)-x_{l}\right\| \leq & \sum_{1 \leq r \leq j}\left\|T_{(j+1) u, \lambda} S_{r u, \alpha_{r-1}}\left(x_{l}\right)\right\| \\
& +\left\|T_{(j+1) u, \lambda} S_{(j+1) u, \alpha_{j}}\left(x_{l}\right)-x_{l}\right\| \\
& +\sum_{j+1<r \leq m}\left\|T_{(j+1) u, \lambda} S_{r u, \alpha_{r-1}}\left(x_{l}\right)\right\| \\
\leq & c_{j u}+\cdots+c_{u}+\frac{1}{2^{l+1}}+c_{u}+\cdots+c_{(m-j-1) u} \\
\leq & 1 / 2^{l},
\end{aligned}
$$

where we used $(\mathrm{CHC})(1)(\mathrm{b}),(\mathrm{CHC})(1)(\mathrm{c})$ and $(\mathrm{CHC})(2)$. This proves (9) for $k=1$. Moreover, (10) is simultaneously true, thanks to the choice of $u$ (see (13)). By the way, for $n \leq \max _{1 \leq j<l, 1 \leq r \leq j} \Gamma_{r, j}$ one has

$$
\begin{aligned}
\left\|T_{n, \lambda} z_{1}(l)\right\| & \leq\left\|T_{n, \lambda} S_{(u-n)+n, \alpha_{0}}\left(x_{l}\right)\right\|+\cdots+\left\|T_{n, \lambda} S_{(m u-n)+n, \alpha_{m-1}}\left(x_{l}\right)\right\| \\
& \leq c_{u-n}+\cdots+c_{m u-n} \leq 1 / 2^{l+1} .
\end{aligned}
$$

So, (12) is proved. By induction, the same construction works for $1 \leq k<l$. The only difference is that we force the integer $u$ to satisfy, in addition to conditions like (13) and (14), the following one:

$$
\forall \lambda \in K_{l}, \forall n \geq u, \forall m<k, \quad \sum_{j \leq l}\left\|T_{n, \lambda} z_{m}(j)\right\|<1 / 2^{k} .
$$

This is always possible, once more thanks to $(\mathrm{CHC})(1)(\mathrm{d})$, and condition (11) is also satisfied. For $k=l$, we slightly diverge by setting

$$
z_{l}(l)=x_{l}+S_{u, \alpha_{0}}\left(x_{l}\right)+\cdots+S_{m u, \alpha_{m-1}}\left(x_{l}\right),
$$

which ensures that (8) is satisfied. The rest of the proof is unchanged.

ExAmple. Let $d \mu$ be the measure $d t /\left(1+t^{2}\right)$ on the real line. For $\lambda>1$, we define the operator

$$
T_{\lambda}: L^{2}(\mathbb{R}, d \mu) \rightarrow L^{2}(\mathbb{R}, d \mu), \quad f(x) \mapsto f(\lambda x) .
$$

It is proved in $[\mathrm{Ba} 2]$ that the family $\left(T_{\lambda}\right)_{\lambda>1}$ satisfies $(\mathrm{CHC})$. It follows from Theorem 7 that there exists a dense subspace of $L^{2}(\mathbb{R}, d \mu)$ consisting entirely, except for 0 , of vectors that are hypercyclic for each $T_{\lambda}$.

Acknowledgements. I thank Prof. V. Nestoridis and Prof. H. Queffélec for stimulating conversations.

\section{References}

[AG] E. Abakumov and J. Gordon, Common hypercyclic vectors for multiples of backward shift, J. Funct. Anal. 200 (2003), 494-504. 
[Bag] B. Bagchi, A joint universality theorem for Dirichlet L-functions, Math. Z. 181 (1982), 319-334.

[Ba1] F. Bayart, Common hypercyclic vectors for composition operators, J. Operator Theory, to appear.

[Ba2] -, Common hypercyclic subspace, Integral Equations Operator Theory, to appear.

[BKQ] F. Bayart, S. Konyagin and H. Queffélec, Convergence almost everywhere and divergence everywhere of Taylor and Dirichlet series, Real Anal. Exchange 29 (2003/2004), 557-586.

[Be] L. Bernal-González, Densely hereditarily hypercyclic sequences and large hypercyclic manifolds, Proc. Amer. Math. Soc. 127 (1999), 3279-3285.

[Bou] P. S. Bourdon, Invariant manifolds of hypercyclic vectors, Proc. Amer. Math. Soc. 118 (1993), 845-847.

[CS] G. Costakis and M. Sambarino, Genericity of wild holomorphic functions and common hypercyclic vectors, Adv. Math. 182 (2004), 278-306.

[Fe] C. Fefferman, On the convergence of multiple Fourier series, Bull. Amer. Math. Soc. 77 (1971), 744-745.

[Gre] K.-G. Grosse-Erdmann, Universal families and hypercyclic operators, Bull. Amer. Math. Soc. 36 (1999), 345-381.

[Ka] J.-P. Kahane, Baire's category theorem and trigonometric series, J. Anal. Math. 80 (2000), 143-182.

[KK] J.-P. Kahane et Y. Katznelson, Sur les ensembles de divergence des séries trigonométriques, Studia Math. 26 (1966), 305-306.

[KN] J.-P. Kahane et V. Nestoridis, Séries de Taylor et séries trigonométriques universelles au sens de Menchoff, J. Math. Pures Appl. 79 (2000), 855-862.

[La] A. Laurinčikas, Limit Theorems for the Riemann Zeta-Function, Kluwer, 1996.

[Ne] V. Nestoridis, Universal Taylor series, Ann. Inst. Fourier 46 (1996), 1293-1306.

[Zyg] A. Zygmund, Trigonometric Series, Cambridge Univ. Press, 1959.

Laboratoire Bordelais d'Analyse et de Géométrie

UMR 5467

Université Bordeaux 1

351 Cours de la Libération

F-33405 Talence Cedex, France

E-mail: bayart@math.u-bordeaux.fr

Received October 7, 2003

Revised version October 20, 2004 